\title{
A Generic Input Device For The Multiple Master Multiple Slave System
}

\author{
Chiun-fan Chen ${ }^{1}$, Yi-chu Chang ${ }^{1}$, Heng-Yin Chen ${ }^{1}$, Jin-shin Lai ${ }^{2}$, Fok-ching Chong ${ }^{1}$ \\ ${ }^{1}$ Department of Electrical Engineering, National Taiwan University, Taipei, Taiwan, R.O.C. \\ ${ }^{2}$ Department of Physical Medicine and Rehabilitation, National Taiwan University Hospital, Taipei, Taiwan, R.O.C.
}

\begin{abstract}
The growing demand for input devices designed for the severely handicapped led to development of modular features for the open architecture Multiple Master Multiple Slave (M3S) system. With its central safety monitor, M3S allows individuals suffering from cerebral palsy, paraplegia, or multiple sclerosis, and other physically debilitating illnesses greater autonomy by allowing them to access a wide range of input devices tailored to a specific user, e.g., joysticks, keypads, head-control modules and speech recognizers. These input devices, which are equipped with basic configuration abilities, are operated with DSP cores and additional circuitries to maintain optimal control and high levels of safety, and may be modified or expanded with minimal adjustments.

Keywords-Multiple Master Multiple Slave, generic input device, Control and Configuration
\end{abstract}

\section{INTRODUCTION}

$\mathrm{R}^{\mathrm{F}}$ ECENT technologies have helped the disabled to overcome physical challenges, leading to significant improvements in their physical, cognitive, commutative, and social skills. In order to meet their varying demands, designers of electronic devices introduced the "modular" featured Multiple Master Multiple Slave (M3S) system.

The main purpose of this paper is to allow easy modification or expansion of a generic input device equipped with basic control and configuration functions of an M3S based system [1-4]. This integrated system provides the option of putting together a variety of electronic modules in the rehabilitation environment, allowing accommodation to different degrees of handicap or whose gradual deterioration of health might require a corresponding increase in system capability (Fig. 1.).

The bus is composed of three components: a controller area network (CAN) bus for digital communication [5], a SAF bus for additional safety and a POW bus for power support. The CAN bus uses a multi-master serial protocol with a very high level of data integrity and sophisticated error detection mechanisms, which permits efficient support of distributed real-time control functions and fast transmission speeds. The SAF bus consists of two signal lines (KEY and DMS) that are coupled to the basic CAN two-line communication channel for extra safety. These lines function independently of the CAN bus and microprocessors in all terminal devices. The battery device supplies power to each device on the M3S bus through the two power lines (POW), namely, the BATT+ and BATT-.

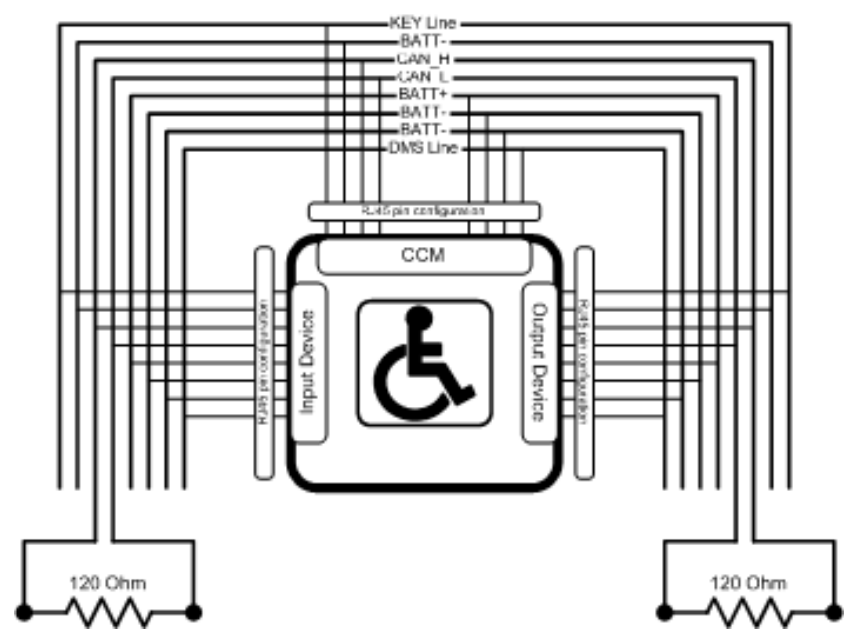

Fig. 1. The System Architecture

\section{Circuitries For Physical Medium Attachment}

Circuitries that allow correct attachment to the M3S Physical Medium are utilized for this application, along with the Texas Instrument TMS320F243 DSP controller as the core processor (Fig. 2.).

1) The Input Module:

Joysticks are often used as the input devices for powered wheelchair systems. They can either be discrete or linear. Like switches, discrete joysticks give digital commands in response to discrete outputs. The output of a linear joystick depends on how far the joystick is pushed away from the center position. A pair of variable resistance is used to signal the position, and a dead-zone is incorporated to differentiate desired input signals from undesired tremor or any other involuntary movement [6].

Most users are able to operate the wheelchair with a joystick, however, for users with severe spasticity or quadriplegic, an alternative control method should be considered, for example, head-control. Commands are sent to the DSP, which generates CAN signals for bus transmission and is received by the target output device. A centrally located dead-zone area allows the user to rest and relax properly after prolonged movement.

Pushing a KEY_ON switch sets the key switch latch, directing BATT+ to supply a limited current through a protection circuitry to the KEY line of the M3S bus. The key switch latch status is read from the KSL_SW_STS signal and isolated from the KEY line so that the $\overline{C C M}$ can detect faults and turn off the system when the KEY line is 


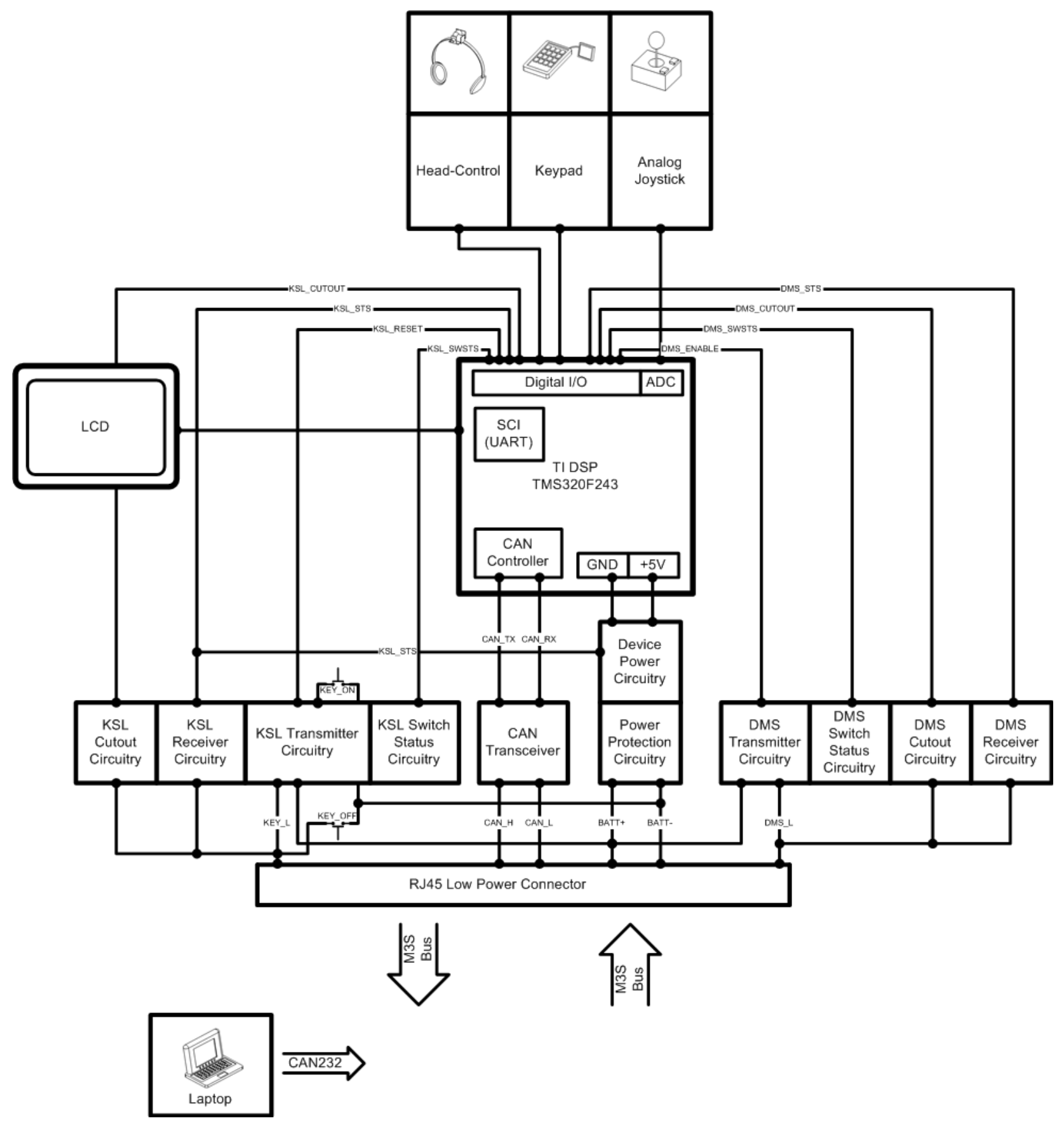

Fig. 2. M3S input device with basic CCM features

short-circuited to BATT+ while no KEY_ON switch is set on any device of the whole system. A C2D_KSL_RESET message is sent through the CAN bus to reset the key switch latch of that certain device. When a KEY OFF switch is set or the KSL_CUTOUT signal is applied, the KEY line is short circuited to BATT-, which calls for the system to shut down. The KEY line makes it possible to turn the whole system on or off by means of any input device with a key switch, however, only one should be active at any time. This allows the user to regain control of the system quickly in the event of a mistake and a helper to turn the system on or off immediately in case of emergency.

The DMS line is activated upon receiving positive feedback in the form of an action. When the action returns to neutral, the DMS line deactivates, cutting off the power supply to safety critical devices such as motor drives. 
2) The Control and Configuration Module (CCM):

Each device on an M3S bus should contain a stored system configuration, which describes the specific behavior of each device in a format that can be understood by the CCM. The CCM also transfers data to and from other devices through the CAN bus. We are working on a preconfigured CCM for a general M3S system with the following features:

1. Task Management: The configuration and control function of the CCM has the proficiency to start different tasks within the M3S system, for example, to operate a wheelchair using voice control. The CCM uploads the configuration information from the devices involved, inputs or outputs are matched with the relevant degrees of freedom (DOFs) and then transferred to data signals that devices could communicate on the M3S bus. The CCM also determines the priority status for each task.

2.Safety Monitoring: The system safety monitor CCM checks for overall safety status and receives confirmation from individual device safety monitors as to whether they are in proper condition. The CCM checks the status of disabled devices once per second and enabled devices each tenth of a second and will take proper action to halt the system when serious faults occur. The CCM also monitors the status and connections of the DMS and KEY lines.

\section{RESULTS}

The nominal bit rate of the CAN bus is $250 \mathrm{kbit} / \mathrm{s}$ (bit time, $\left.N_{N B T}=4.0 \mu \mathrm{s}\right)$, resynchronization jump width is three quanta long $\left(N_{S J W}=1.5 \mu \mathrm{s}\right)$ and the sample point is specified at $5 / 8$ of the nominal time $\left(N_{T S E G 2}=1.5 \mu s\right)$. The maximum oscillator tolerance can be calculated as follows [5]:

Max. Osc. Tolerance $=\frac{N_{S J W}}{2 \cdot\left(30 \cdot N_{N B T}-N_{\text {TSEG } 2}\right)}=0.633 \%$

Signaling and attachment of the CAN bus was monitored with HP54603B and a LAWICEL CAN232 dongle (Modified under permission from CAN232 MONITOR by John Dammeyer, COM port Library written by Dejan Crnila) (Fig. 3.). Data transfer of several M3S messages were validated with CAN bus transmissions.

TABLE I

\begin{tabular}{llll}
\hline \multicolumn{4}{l}{ M3S MESSAGE TRANSFER ON CAN BUS } \\
\cline { 2 - 4 } & NAME & CAN ID & DATA [0] \\
\hline 1 & EMERGENCY_STOP & 0x000 & - \\
2 & SYSTEM_RESET & $0 \times 001$ & - \\
3 & C2D_STOP & $0 \times 101$ & 0x00 \\
4 & C2D_RESET & $0 \times 101$ & 0x01 \\
5 & C2D_SET_DOF & 0x101 & 0x0E \\
6 & D2C_DISCONNECT & 0x181 & 0x00 \\
\hline
\end{tabular}
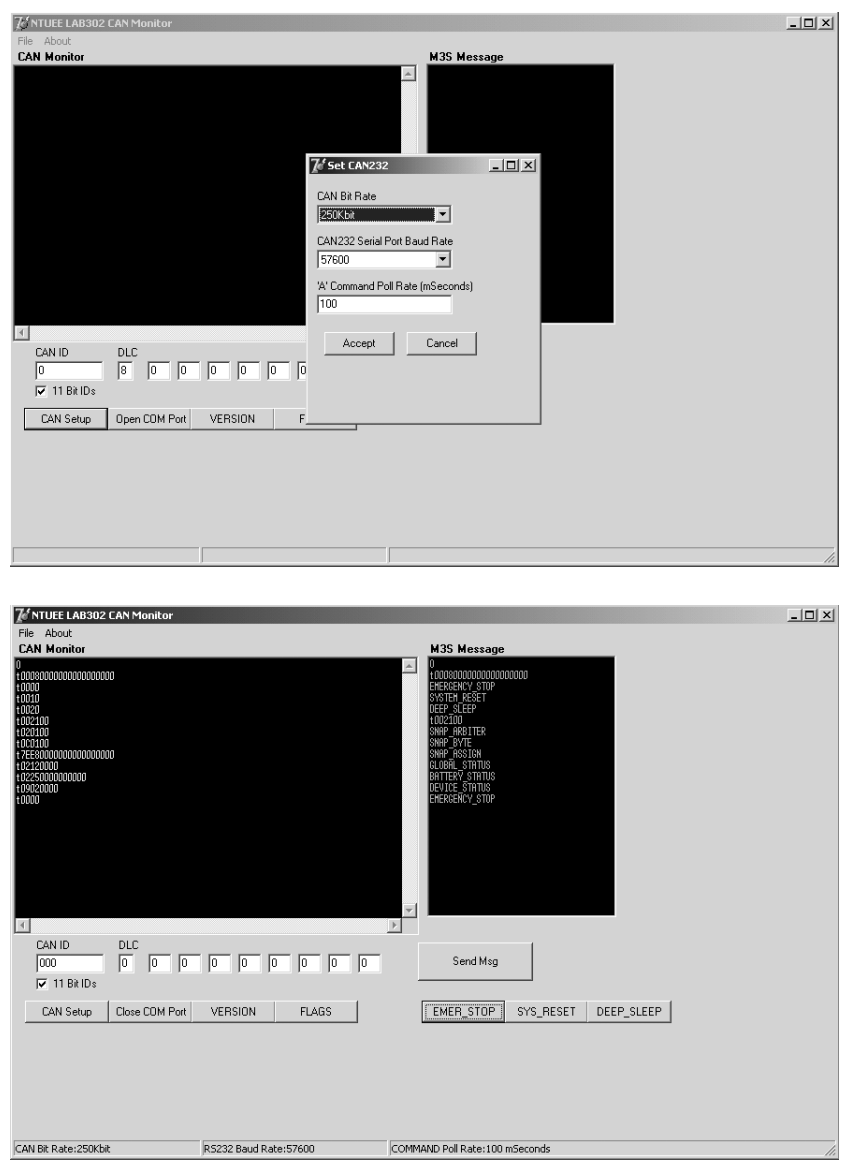

Normal Transmission

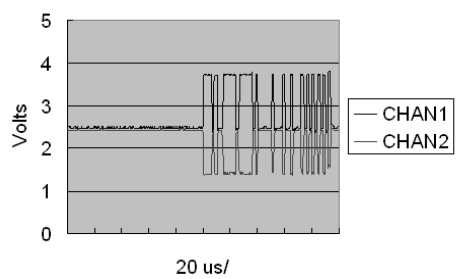

Error Transmission

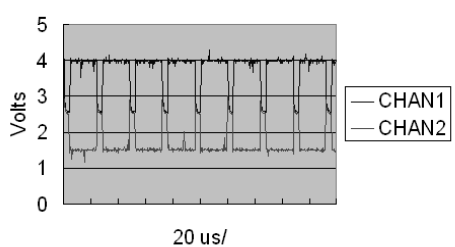

Fig. 3. Monitoring CAN transmissions

The physical layer compliance of the KEY and DMS lines involved minimum active voltage, maximum release voltage, maximum off voltage, maximum cut out voltage 
drop and the minimum/maximum active limit current tests and met the standards of the M3S protocol. The minimum active voltage (7Volts) and maximum release voltage (4Volts) of the KEY (or DMS) line controls the logic of the KEY (or DMS) status voltage, which is clamped to 0 or 5 Volts for DSP interface. Results of the Electromagnetic Interference (EMI) test are shown on Fig. 4.

\section{CONCLUSION}

A prototype is designed with the objective of building a versatile base model to which modules can be added or removed by individuals with minimal technical expertise. Users that suffer from greater degrees of disability are more likely to use input systems that allow discrete commands to be issued via a tree menu, which provides greater control over the user interface. These may be designed by means of a generic device without complicated adaptations. Userdependent isolated word recognition is under development with a DSP core.

The system allows for addition of new applications through wireless links and can easily be utilized to maintain a domotic network.
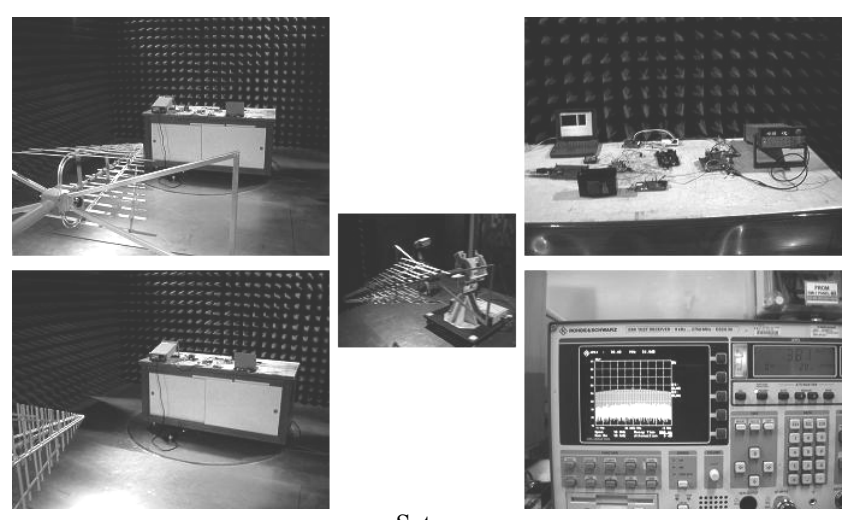

Setup

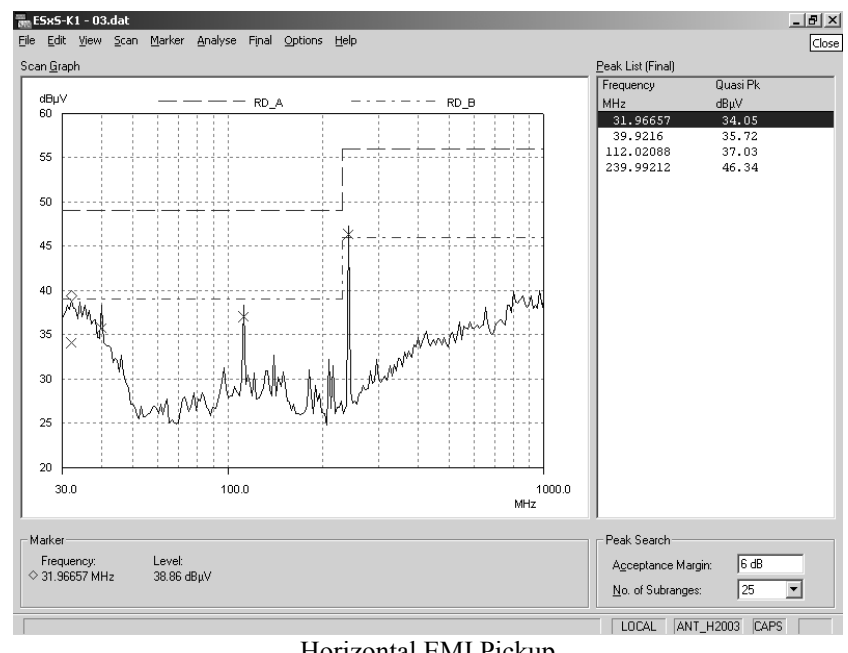

Horizontal EMI Pickup

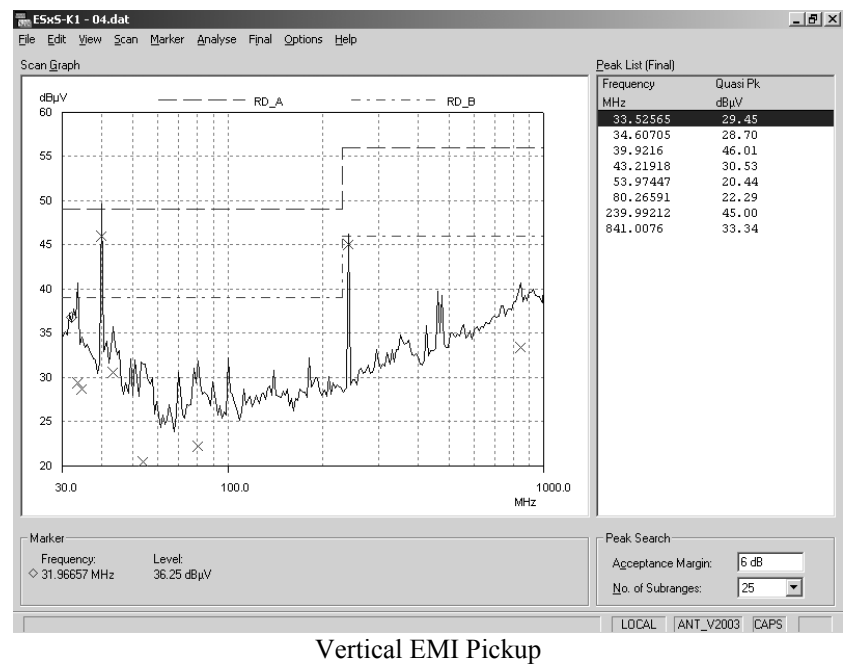

Fig. 4. Electromagnetic Interference test

\section{ACKNOWLEDGMENT}

This work was supported by the National Science Council, Taiwan, R.O.C. under grant NSC-92-2218-E002022

\section{REFERENCES}

[1] M3S Reference Manual, Version 2.00-revision 9, July 1997. Available: http://www.tno.nl/m3s/

[2] S. Linnman, "M3S: The Local Network for Electric Wheelchairs and Rehabilitation Equipment," IEEE Trans. on Rehab. Eng. 4(3), pp. 188-192 Sept. 1996

[3] B. Davies, R. Hibberd, "A Safe Communication System for Wheelchair-Mounted Medical Robots," Comput. \& Contrl. Eng. J. 6(5), pp. $216-221$, Oct. 1995

[4] J. A. van Woerden, M. W. Nelisse, C. Perricos, R. D. Jackson, B Davies, R. D. Hibberd, D. Banerjee, "M3S-A Standard Communication Architecture for Rehabilitation Applications," Comput. \& Contrl. Eng. J. 5(5), pp. $213-218$, Oct. 1994

[5] CAN Specification, Version 2.0, ROBERT BOSCH GmbH, 1991. [6] R. A. Cooper, "Intelligent Control of Power Wheelchairs," IEEE Eng. in Med. \& Bio. Mag. 14(4), pp. 423-431, 1995 